\title{
Three-Dimensional Medical Image Recognition of Blood Vessels by Multi-Layered GMDH-Type Neural Network Self-Sellecting Optimum Neural Network Architecture
}

\author{
Tadashi Kondo \\ School of Health Sciences, The University of Tokushima,3-18-15 \\ Kuramoto-cho Tokushima 770-8509, Japan \\ kondo@medsci.tokushima-u.ac.jp
}

\begin{abstract}
In this study, a new multi-layered Group Method of Data Handling (GMDH)-type neural network self-selecting optimum neural network architecture is proposed. We call this algorithm as revised GMDH-type neural network algorithm self-selecting optimum neural network architecture. Revised GMDH-type neural network algorithm has an ability of self-selecting optimum neural network architecture from three neural network architectures such as sigmoid function neural network, radial basis function (RBF) neural network and polynomial neural network. Revised GMDH-type neural network also have abilities of self-selecting the number of layers, the number of neurons in hidden layers and useful input variables. This algorithm is applied to medical image recognition and it is shown that this algorithm is useful for medical image recognition and is very easy to apply practical complex problem because optimum neural network architecture is automatically organized.
\end{abstract}

\section{Introduction}

Group Method of Data Handling (GMDH)-type neural networks and their applications have been proposed in our early works [1],[2]. GMDH-type neural networks can automatically organize neural network architecture by heuristic self-organization method [3],[4] and they can also determine such structural parameters as the number of layers, the number of neurons in hidden layers and useful input variables. But, these conventional GMDH-type neural network algorithms do not have an ability of selecting the type of neural network architectures such as sigmoid function neural network, radial basis function (RBF) neural network and polynomial neural network.

In this study, a revised GMDH-type neural network algorithm self-selecting optimum neural network architecture is proposed. In this algorithm, optimum neural network architecture is automatically selected from three neural network architectures such as sigmoid function neural network, RBF neural network and polynomial neural network. Furthermore, structural parameters such as the number of layers, the number of neurons in hidden layers and useful input variables are automatically selected to minimize prediction error criterion defined as Prediction Sum of Squares (PSS)[5]. Revised GMDH-type neural network algorithm proposed in this paper is applied to medical image recognition and results show that revised GMDH-type neural network algorithm is useful for medical image recognition and is very easy to apply practical complex problem because optimum neural network architecture is automatically organized.

\section{GMDH-Type Neural Network}

Architectures of GMDH-type neural network are automatically organized by heuristic self-organization method. First, we show procedures of heuristic self-organization method because it plays very important roles for organization of GMDH-type neural network

\subsection{Heuristic Se1f-Organization}

Heuristic self-organization method is constructed by the following six procedures:

(1) Separating original data into training and test sets

Original data is separated into training and test sets. Training data is used for estimating parameters of partial descriptions which describe partial relationships of the nonlinear system. Test data is used for organizing complete description which describes complete relationships between input and output variables of the nonlinear system.

(2) Generating combinations of input variables in each layer

All combinations of two input variables $\left(x_{i}, x_{j}\right)$ are generated in each layer. The number of combinations is $\frac{p !}{(p-2) ! 2 !}$. Here, $p$ is the number of input variables.

(3) Calculating partial descriptions

For each combination, partial descriptions of the nonlinear system can be calculated by applying regression analysis to training data. Output variables of partial descriptions are called as intermediate variables.

(4) Selecting intermediate variables

$L$ intermediate variables which give $L$ smallest test errors calculated using test data are selected from generated 
intermediate variables.

\section{(5) Iterating calculations from 2 to 5}

Select $L$ intermediate variables are set to input variables of the next layer and calculations from procedure 2 to 5 are iterated. The multi-layered architecture is organized.

(6) Stopping multilayered iterative calculation

When errors of test data in each layer stop decreasing, iterative calculation is terminated. Finally, complete description of the nonlinear system is constructed by partial descriptions generated in each layer.

\subsection{Revised GMDH-Type Neural Network Algorithm}

Revised GMDH-type neural network has a common feedforward multilayered architecture. Figure 1 shows architecture of revised GMDH-type neural network. This neural network is organized by heuristic self-organization method.

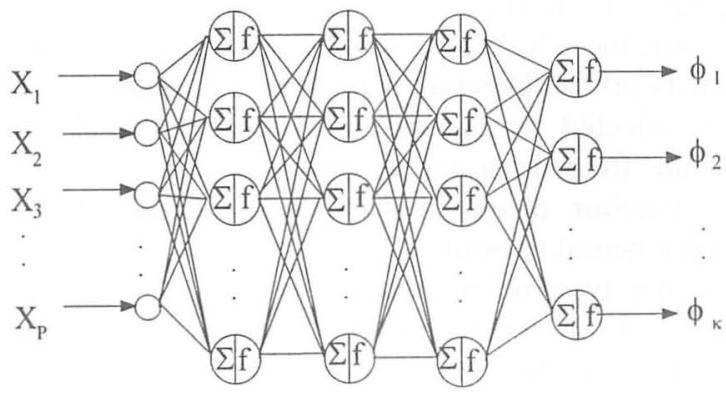

Fig.1 Architecture of revised GMDH-type neural network

Procedures for determining architecture of revised GMDH-type neural network conform to the following:

(1) The first layer

$u_{j}=x_{j}(j=1,2, \ldots, p)$

where $x_{j}(j=1,2, \ldots, p)$ are input variables of the nonlinear system, and $p$ is the number of input variables. In the first layer, input variables are set to output variables.

(2) The second layer

All combinations of $r$ input variables are generated. For each combination, optimum neuron architectures are automatically selected from the following two neurons.

Architectures of the first and second type neurons are shown in Fig. 2 and Fig. 3 respectively. Optimum neuron architecture for each combination is selected from the first and second type neuron architectures. $\mathrm{u}_{\mathrm{i}}$

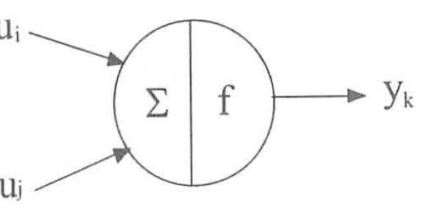

Fig.2 Neuron architecture with two inputs

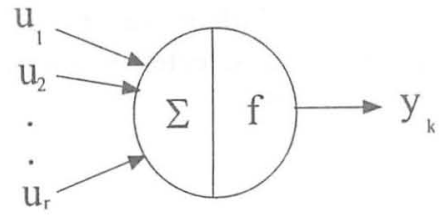

Fig.3 Neuron architecture with $r$ inputs
Revised GMDH-type neural network algorithm proposed in this paper can select optimum neural network architecture from three neural network architectures such as sigmoid function neural network, RBF neural network and polynomial neural network.

Neuron architectures of the first and second type neurons in each neural network architecture are shown as follows.

$<$ Sigmoid function neural network $>$

i) The first type neuron

$\Sigma$ : (Nonlinear function)

$z_{k}=w_{1} u_{i}+w_{2} u_{j}+w_{3} u_{i} u_{j}+w_{4} u_{i}^{2}+w_{5} u_{j}^{2}+w_{6} u_{i}^{3}+w_{7} u_{i}^{2} u_{j}+w_{8} u_{i} u_{j}^{2}$

$+w_{9} u_{j}^{3}-w_{0} \theta_{l}$

$\mathrm{f}:$ (Nonlinear function)

$y_{k}=\frac{1}{1+e^{\left(-z_{k}\right)}}$

Here, $\theta_{1}=1$ and $w_{i}(i=0,1,2, \ldots, 9)$ are weights between the first and second layer. Value of $r$, which is the number of input variables $u$ in each neuron, is set to two for the first type neuron.

ii) The second type neuron

$\Sigma$ : (Linear function)

$z_{k}=w_{l} u_{l}+w_{2} u_{2}+w_{3} u_{3}+\cdots+w_{r} u_{r}-w_{0} \theta_{l} \quad(r<p)$

$\mathrm{f}$ : (Nonlinear function)

$y_{k}=\frac{1}{1+e^{\left(-z_{k}\right)}}$

Here, $\theta_{1}=1$ and $w_{i}(i=0,1,2, \ldots, r)$ are weights between the first and second layer. Value of $r$, which is the number of input variables $u$ in each neuron, is set to be greater than two and smaller than $p$ for the second type neuron. Here $p$ is the number of input variables $x_{i}(i=1,2, \ldots, p)$.

$<$ Radial basis function neural network $>$

i) The first type neuron

$\Sigma:$ (Nonlinear function)

$z_{k}=w_{1} u_{i}+w_{2} u_{j}+w_{3} u_{i} u_{j}+w_{4} u_{i}^{2}+w_{5} u_{j}^{2}+w_{6} u_{i}^{3}+w_{7} u_{i}^{2} u_{j}+w_{8} u_{i} u_{j}^{2}$

$+w_{9} u_{j}^{3}-w_{0} \theta_{l}$

$\mathrm{f}:$ (Nonlinear function)

$y_{k}=e^{\left(-z_{k}^{2}\right)}$

Here, $\theta_{1}=1$ and $w_{i}(i=0,1,2, \ldots, 9)$ are weights between the first and second layer. Value of $r$, which is the number of input variables $u$ in each neuron, is set to two for the first type neuron.

ii) The second type neuron

$\Sigma$ : (Linear function)

$z_{k}=w_{1} u_{l}+w_{2} u_{2}+w_{3} u_{3}+\cdots+w_{r} u_{r}-w_{0} \theta_{l} \quad(r<p)$

$\mathrm{f}:$ (Nonlinear function)

$y_{k}=e^{\left(-z_{k}^{2}\right)}$

Here, $\theta_{l}=1$ and $w_{i}(i=0,1,2, \ldots, r)$ are weights between the first and second layer. Value of $r$, which is the number of input variables $u$ in each neuron, is set to be greater than two and smaller than $p$ for the second type neuron. Here $p$ is the number of input variables $x_{i}(i=1,2, \ldots, p)$.

$<$ Polynomial neural network $>$

i) The first type neuron 
$\Sigma:$ (Nonlinear function)

$$
\begin{aligned}
& z_{k}=w_{I} u_{i}+w_{2} u_{j}+w_{3} u_{i} u_{j}+w_{4} u_{i}^{2}+w_{5} u_{j}^{2}+w_{6} u_{i}^{3}+w_{7} u_{i}^{2} u_{j}+w_{8} u_{i} u_{j}^{2} \\
& +w_{9} u_{j}^{3}-w_{0} \theta_{l} \\
& \mathrm{f} \text { : (Linear function) } \\
& y_{k}=z_{k}
\end{aligned}
$$

Here, $\theta_{l}=1$ and $w_{i}(i=0,1,2, \ldots, 9)$ are weights between the first and second layer. Value of $r$, which is the number of input variables $u$ in each neuron, is set to two for the first type neuron.

ii) The second type neuron

$\Sigma$ : (Linear function)

$z_{k}=w_{1} u_{l}+w_{2} u_{2}+w_{3} u_{3}+\cdots+w_{r} u_{r}-w_{0} \theta_{l} \quad(r<p)$

$\mathrm{f}:$ (Linear function)

$y_{k}=z_{k}$

Here, $\theta_{1}=1$ and $w_{i}(i=0,1,2, \ldots, r)$ are weights between the first and second layer. Value of $r$, which is the number of input variables $u$ in each neuron, is set to be greater than two and smaller than $p$ for the second type neuron. Here $p$ is the number of input variables $x_{i}(i=1,2, \ldots, p)$.

Weights $w_{i}(i=0,1,2, \ldots)$ in each neural network architecture are estimated by stepwise regression analysis using PSS.

\section{[Estimation procedure of weight $w_{i}$ ]}

First, values of $z_{k}$ are calculated for each neural network architecture as follows.

i) Sigmoid function neural network

$z_{k}=\log _{e}\left(\frac{\phi^{\prime}}{1-\phi^{\prime}}\right)$

ii) $\mathrm{RBF}$ neural network

$$
z_{k}=\sqrt{-\log _{e} \phi^{\prime}}
$$

iii) Polynomial neural network

$$
z_{k}=\phi
$$

where $\phi^{\prime}$ is normalized output variable whose values are between zero and one and $\phi$ is output variable.

Then weights $w_{i}$ are estimated by stepwise regression analysis [6] which selects useful input variables using PSS. Only useful variables in Eq.(2), Eq.(4), Eq.(6), Eq.(8), Eq.(10) and Eq.(12) are selected by stepwise regression analysis using PSS and optimum neuron architectures are organized by selected useful variables.

PSS is described by the following equation:

$P S S=\sum_{\alpha=1}^{n}\left(\phi_{\alpha^{-}} y_{\alpha}^{*}\right)^{2}$

where

$y_{\alpha}{ }^{*}=b_{0 \alpha}+b_{1 \alpha} x_{i \alpha}+b_{2 \alpha} x_{j \alpha}+b_{3 \alpha} x_{i \alpha} x_{j \alpha}+b_{4 \alpha} x_{i \alpha}{ }^{2}+b_{5 \alpha} x_{j \alpha}{ }^{2}$,

$$
\alpha=1,2, \ldots, n
$$

$n$ is the number of training data, $\phi_{\alpha}$ is the $\alpha$-th observed value for the output variable, $x_{i \alpha}$ is the $\alpha$-th observed value for the input variable $x_{i}$ and $y_{\alpha}{ }^{*}$ is the $\alpha$-th estimated value obtained by the multiple regression analysis of all the data except the $\alpha$-th datum. In order to calculate PSS in (17), the multiple regression analysis must be repeated $n$ times, and the amount of calculations increases in the number of data. But PSS in (17) can be reduced as follows,

$$
P S S=\sum_{\alpha=1}^{n} \frac{\phi_{\alpha}-y_{\alpha}}{\left(1-\underline{x}_{\alpha}^{T}\left(X^{T} X\right)^{-1} \underline{x}_{\alpha}\right)^{2}}
$$

where

$y_{\alpha}=b_{0}+b_{1} x_{i \alpha}+b_{2} x_{j \alpha}+b_{3} x_{i \alpha} x_{j \alpha}+b_{4} x_{i \alpha}{ }^{2}+b_{5} x_{j \alpha}{ }^{2}$,

$\underline{x}_{\alpha}{ }^{T}=\left[1, x_{i \alpha}, x_{j \alpha}, x_{i \alpha} x_{j \alpha}, x_{i \alpha}{ }^{2}, x_{j \alpha}{ }^{2}\right], \quad \alpha=1,2, \ldots, n$

$\mathrm{X}^{\mathrm{T}}=\left[\underline{x}_{1}, \underline{x}_{2}, \ldots, \underline{x}_{n}\right]$

$y_{\alpha}$ is the $\alpha$-th estimated value obtained by the multiple regression analysis of all the data. With this procedure, we need not repeat the regression analysis $n$ times. PSS criterion do not contain the statistical assumption in the regression model.

For each combination, three neuron architectures which are sigmoid function neuron, RBF neuron and polynomial neuron, are generated and $L$ neurons which minimize test error calculated using test data are selected for each neuron architecture. From these $L$ selected neurons for each neuron architecture, mean test errors of $L$ neurons are calculated. Then, neural network architecture which has minimum mean test error is selected as revised GMDH-type neural network architecture from three neural network architectures such as sigmoid function neural network, RBF neural network and polynomial neural network.

After the type of revised GMDH-type neural network architecture is selected, output variables $y_{k}$ of $L$ selected neurons are set to input variables of neurons in the third layer.

\section{(3) The third and successive layers}

In the second layer, optimum neural network architecture is selected from three neural network architectures. In the third and successive layers, only one neuron architecture, which is sigmoid function neuron or RBF neuron or polynomial neuron, is used for calculation and the same calculation of the second layer is iterated until PSS values of $L$ neurons with selected neuron architecture stop decreasing. When iterative calculation is terminated, neural network architecture is produced by $L$ selected neurons in each layer.

By using these procedures, revised GMDH-type neural network self-selecting optimum neural network architecture is organized. Figure 4 shows the flowchart of the revised GMDH-type neural network. Revised GMDH-type neural network proposed in this paper has an ability of self-selecting optimum neural network architecture. Therefore, neural network architecture is automatically selected from three neural network architectures. Furthermore, structural parameters such as the number of layers, the number of neurons in hidden layers and useful input variables are automatically selected to minimize prediction error criterion defined as PSS. 


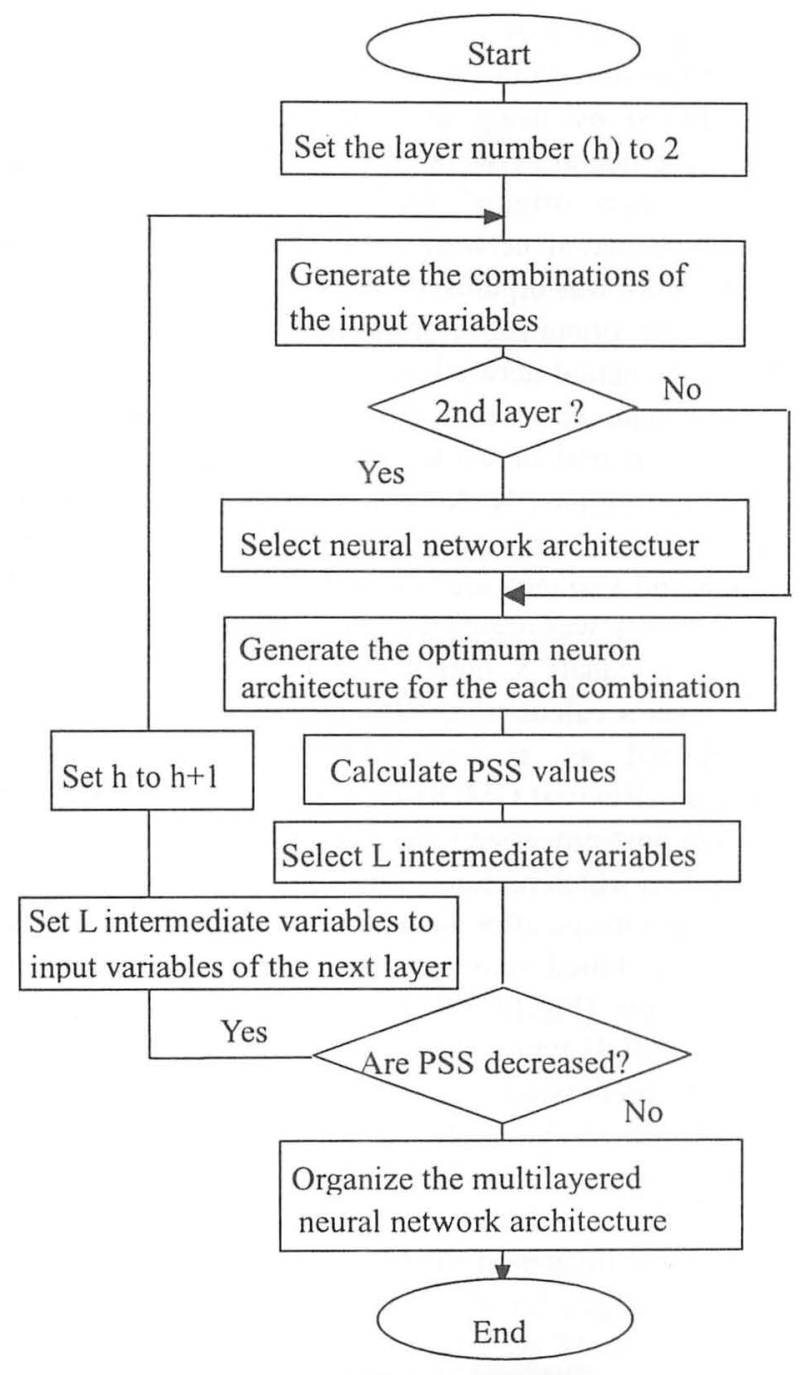

Fig.4 Flowchart of revised GMDH-type neural network

\section{Application to 3-Dimensional Medical Image Recognition of the Blood Vessels of the Liver}

In this study, regions of the blood vessels of the liver is recognized automatically using the following two recognition procedures. Multi-detector row CT (MDCT) images of the liver are used in this study. In the first recognition procedure, the revised GMDH-type neural network is organized to recognize the liver regions and then these regions are extracted using organized neural network. In the second recognition procedure, another new revised GMDH-type neural network is organized to recognize the blood vessel regions and then these regions are extracted using organized new neural network. Using these recognition procedures, the blood vessel regions are recognized and extracted.

\subsection{Recognition of Liver Regions}

In this study, an original image shown in Fig. 5 is used for organizing revised GMDH-type neural network. Then, image features are extracted and used as input variables of neural network. Statistics of image densities in neighboring regions, $\mathrm{N} \times \mathrm{N}$ pixel regions, are used as image features. The following statistics are used as input variables. 1) mean, 2) standard deviation, 3) variance, 4) median, 5) minimum, 6) maximum, 7) range. Out of these statistics, only three parameters namely, mean, standard deviation and variance are selected as useful input variables. Output value of neural network is zero or one. When $N \times N$ pixel region is contained in regions of the liver, neural network sets pixel value at the center of $N \times N$ pixel region to one and this pixel is shown as white point. Neural network was organized when values of $\mathrm{N}$ are 5,10 and 15 . When $\mathrm{N}$ equals 5 , output image is most accurate. Calculation of revised GMDH-type neural network was terminated at the fourth layer. Three useful neurons were selected in each hidden layer. In the second layer's calculation, RBF neural network architecture was selected as revised GMDH-type neural network architecture. In Fig.6, test errors of three selected neurons in the second layer are shown. Test errors of RBF neurons are smallest in three neuron architectures which are sigmoid function neuron, RBF neuron and polynomial neuron. Revised GMDH-type neural network output liver image and post-processing analysis of liver image was carried out, based on which regions of the liver were extracted. In post-processing of output image of the neural network, small isolated regions outside or inside of the liver regions are eliminated by the image processing such as the dilatation and the erosion. Then, outlines of regions of the liver were expanded outside by $\mathrm{N} / 2$ pixels and outline of the liver were extracted. Fig. 7 shows output image after this processing. In order to check matching between original image and output image of the neural network, the output image was overlapped on original image after post-processing. Overlapped image is shown in Fig.8. From Fig.8, we can see that extracted regions are very accurate.

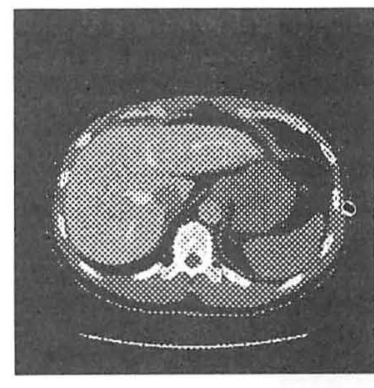

Fig.5 Original image(1)

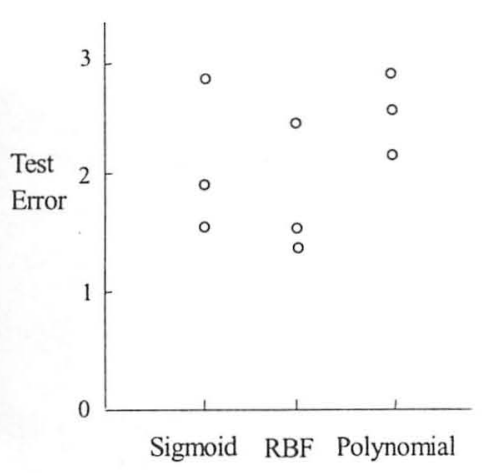

Fig.6 Test errors of three neurons 


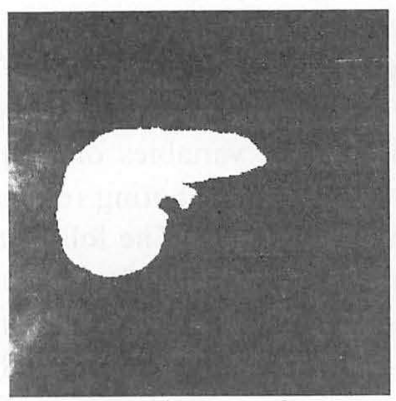

Fig.7 Output image after post-processing (1)

\subsection{Check of Generalization of Neural Network}

In order to check generalization of revised GMDH-type neural network, revised GMDH-type neural network, which was organized by original image of the liver (Fig.5), is applied to another original image of the liver (Fig.9). Revised GMDH-type neural network output the liver image and post-processing analysis of the liver image was carried out, based on which regions of the liver were extracted. The outline of regions of the liver were expanded outside by N/2 pixels. Fig. 10 shows the output image after the post-processing. In order to check matching between the original image and the output image of the neural network, the output image was overlapped on the original image after the post-processing. Overlapped image is shown in Fig.11. From Fig.11, we can that revised GMDH-type neural network could extract new regions of the liver accurately and it is shown that revised GMDH-type neural network has a good generalization ability.

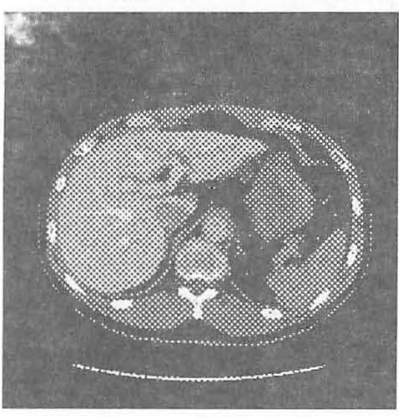

Fig.9 Original image (2)

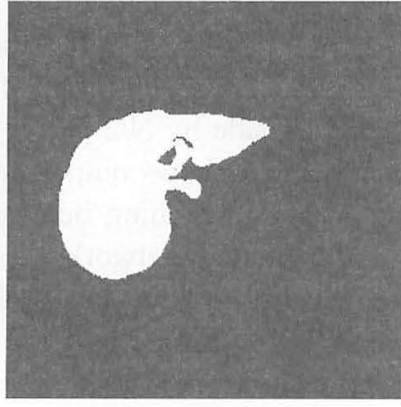

Fig.10 Output image after post-processing (2)

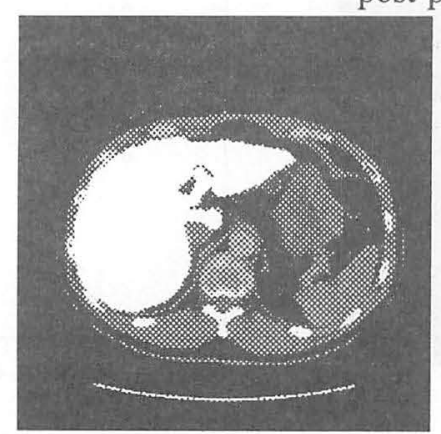

Fig. 11 Overlapped image (2)
3.3 Recognition of Blood Vessel Regions in the Livers

The blood vessel regions are recognized by the revised
GMDH-type neural network and extracted. First, gray scale image of the liver (Fig.12) was subtracted from the original image (Fig.5) by using the output image of the revised GMDH-type neural network (Fig.7). This gray scale image is used as a new original image to organize new revised GMDH-type neural network. The new revised GMDH-type neural network was organized and recognized the blood vessel regions. The organization procedures of the new revised GMDH-type neural network are the same as those of the liver regions. Image features are extracted and used as input variables of neural network. Statistics of image densities in neighboring regions, $\mathrm{N} \times \mathrm{N}$ pixel regions, are used as image features. Only three parameters namely, mean, standard deviation and variance are selected as useful input variables. Neural network was organized when values of $\mathrm{N}$ are 5, 10 and 15. When $\mathrm{N}$ equals 5 , output image is most accurate. In the second layer's calculation, RBF neural network architecture was selected as revised GMDH-type neural network architecture. Revised GMDH-type neural network output liver image and post-processing analysis of liver image was carried out, based on which regions of the liver were extracted. Fig.13 shows output image after the post-processing. Then, gray scale image of the blood vessel (Fig.14) was subtracted from the original image (Fig.12) by using the output image of the revised GMDH-type neural network (Fig.13). These subtraction processing were carried out for the all slices of MDCT. Figure 15 shows another subtraction image of the liver and Fig. 16 shows output image after the post-processing and Fig.17 shows subtraction gray scale image. Then, 3-dimensional images of the liver were generated using these gray scale images for all slices of MDCT by the rendering software. Figure 18 are 3 -dimensional blood vessel images.

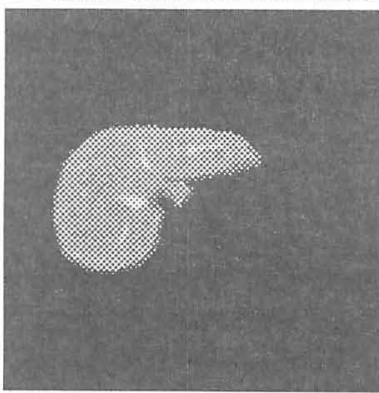

Fig.12 Subtraction image of the liver (1)

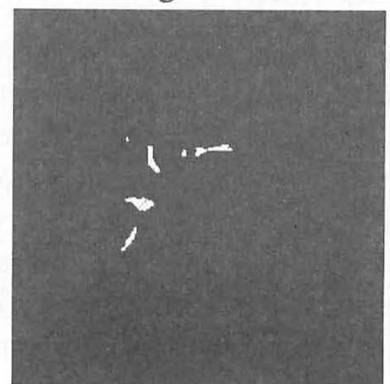

Fig.13 Recognition of blood Vessels regions (1)

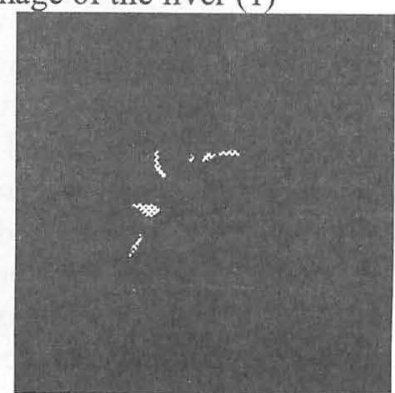

Fig.14 Subtraction of blood vessels regions (1) 


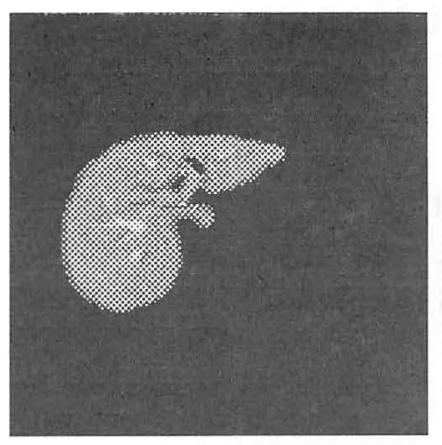

Fig.15 Subtraction image of the liver (2)

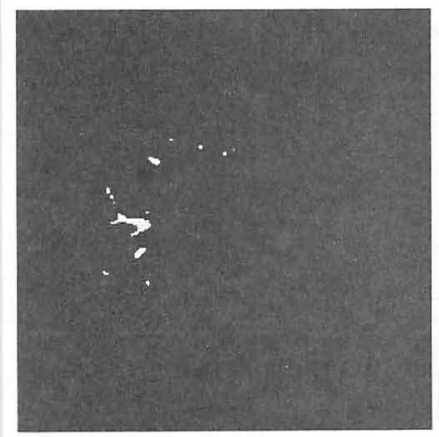

Fig.16 Recognition of blood Fig.17 Subtraction of blood Vessels regions (2)

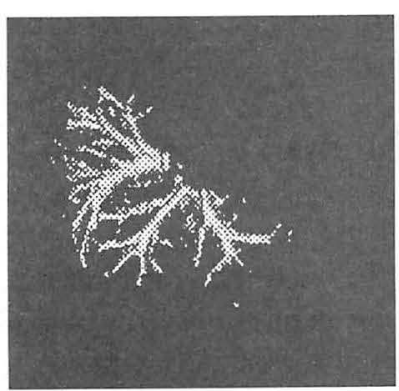

(a) Low

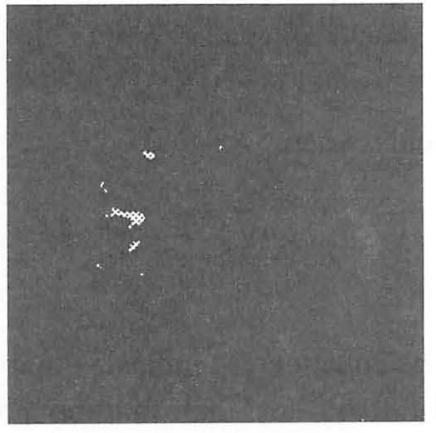
vessels regions (2)

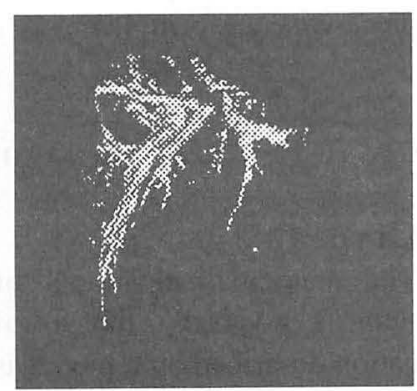

(b) Front

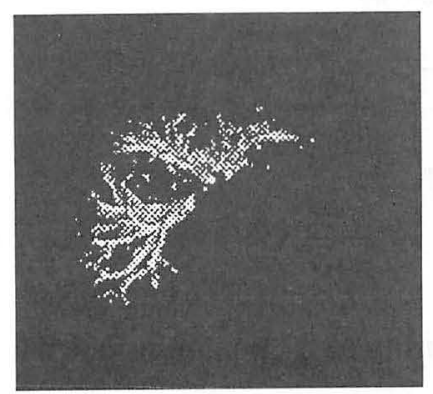

(c) Up

Fig.18 3-dinensional image of the blood vessels

\section{Conclusion}

In this paper, revised GMDH-type neural network algorithm self-selecting optimum neural network architecture was proposed and 3-dimensional liver images were generated by this revised GMDH-type neural networks. In this algorithm, optimum neural network architecture is automatically selected from three neural network architectures such as sigmoid function neural network, RBF neural network and polynomial neural network. Furthermore, structural parameters such as the number of layers, the number of neurons in hidden layers and useful input variables are automatically selected to minimize prediction error criterion defined as PSS.

In this paper, this algorithm was applied to 3-dimensional medical image recognition of the liver and it was shown that revised GMDH-type neural network algorithm was a useful method for 3-dimensional medical image recognition of the liver because the neural network architecture is automatically organized by the revised GMDH-type neural network algorithm.

\section{References}

[1] T.Kondo: GMDH neural network algorithm using the heuristic self-organization method and its application to the pattern identification problem, Proc. of the 37th SICE Annual Conference, pp.1143-1148, 1998.

[2] T.Kondo: Revised GMDH-type neural networks using prediction error criterions AIC and PSS, Proc. of SCIS \& ISIS 2004, WP-6-4, pp.1-6, 2004.

[3] S.J.Farlow ed.: Self-organizing methods in modeling, GMDH-type algorithm, Marcel Dekker, Inc., New York, 1984.

[4] A.G.Ivakhnenko: Heuristic self-organization in problems of engineering cybernetics, Automatica, Vo.6, No.2, pp.207-219, 1970.

[5] H.Tamura and T.Kondo: Heuristics free group method of data handling algorithm of generating optimum partial polynomials with application to air pollution prediction, Int. J. System Sci., Vol.11, No.9, pp.1095-1111, 1980.

[6] N.R.Draper and H.Smith: Applied Regression Analysis, John Wiley and Sons, New York, 1981. 